\title{
Early Feeding vs 5-Day Fasting after Distal Elective Bowel Anastomoses in Children. A Randomized Controlled Trial
}

\author{
Roberto Davila-Perez, Eduardo Bracho-Blanchet, Francisco Galindo-Rocha, \\ Jose Tovilla-Mercado, Gustavo Varela-Fascinetto, Emilio Fernandez-Portilla, \\ Pablo Lezama-del-Valle, Jaime Nieto-Zermeño \\ Pediatric Surgery Department, Hospital Infantil de Mexico Federico Gomez, Mexico City, Mexico \\ Email: robdape001@gmail.com
}

Received October 4, 2012; revised November 2, 2012; accepted November 11, 2012

\begin{abstract}
Purpose: Determine the safety and efficacy of early enteral feeding after distal elective bowel anastomoses (DEBA) in children. Methods: Controlled randomized trial including pediatric patients with DEBA, excluding non-elective and high risk patients. Variables: Demographics, operative time, anastomosis placement, beginning peristalsis and bowel movement, time to full diet intake, post-operative stay, persisting vomiting and abdominal distention, wound infection or dehiscence, anastomotic leak, reoperation, death. Randomization into: 1) Experimental group (EG): early feeding group, after a minimum 24 hours fasting period, oral fluids and diet was started; 2) Control group (CG): obligatory 5-day fasting. Descriptive Statistics: Student's t test for quantitative and Chi square for qualitative variables, a p-value < 0.05 was considered significant. Results: 60 patients were included since June 2003 to May 2004, 30 in each group. Mean age 2 years, weight $16 \mathrm{~kg}$, malnutrition 33\%. Stomal Ethiology: Anorectal-malformation 46\%, Hirschsprung 13\%, inflammatory 35\%, tumoral 5\%. Mostly in colon 71\%. Mean surgical time $142 \mathrm{~min}$. None developed vomiting or required nasogastric-tube. Mild abdominal distension 13\%, mild fever $16.5 \%$ and wound complications $18 \%$. Anastomosis leakage 6.5\%, none required reoperations. Demographic variables showed no statistical differences. Full oral intake was in the 2nd postoperative day in the EG vs CG (p = 0.001). Postoperative hospital stay was $6.0 \pm 3$ in the EG vs 9.8 \pm 4 days in the CG with clinical but not statistical significance. Peristalsis beginning, first flatus passage and bowel movements showed no statistical differences. The complication incidence was low and equally distributed. Conclusions: Early feeding after DEBA is safe and well tolerated in children.
\end{abstract}

Keywords: Early Feeding; Bowel Anastomosis; Children

\section{Introduction}

After the demonstration that nasogastric tube (NGT) decompression was not necessary after a distal elective bowel anastomosis (DEBA) [1], in the last few years the standard management after DEBA at our hospital became a mandatory 5-day fasting without the use of a NGT. This was justified by the perception that the fasting would protect the anastomosis from any complication such as abdominal distention, vomiting, ileus, anastomotic dehiscence or leaks, wound infections and would allow a hermetic closure of the anastomosis before the beginning of the enteral feeding [2-4]. However, there are not any scientific bases for these ideas and the effect of a prolonged fasting in DEBA in children has not been well analyzed. On the other hand, there are a lot of justified reasons to believe that it is functionally possible to begin early feeding (EF), before the postoperative (POP) day 5 in this setting: 1) Clinical and electrophysiological studies have shown that the small bowel recovers normal function within 4 - 8 hours of laparotomy and the colon within 24 hours; 2) The electrolytic, glucose and nutrient absorption ability of the bowel is not affected after a DEBA [3,4]; 3) It is clearly demonstrated that the mucosal epithelium of the bowel is perfectly sealed after the first 24 hours of the POP period [3,4]; 4) Early feeding accelerates the wound and anastomosis healing in the animal model [5]; and 5) There is clear evidence that EF is associated with less incidence of nosocomial infections, liver dysfunction, POP stay, bacterial translocation, secondary malnutrition, and promotes peristalsis, bowel movements and ambulation in surgical adult patients [3,4,6-11]. Pearl et al. demonstrated that EF is safe and well tolerated in gynecologic and oncology adult patients but included both, surgeries with and without opening the bowel [12]. There is only one study in 34 children postoperated (PO) of colostomy closure secondary to anorectal malformation in which the authors concluded that the EF is safe and allows less hospital stay but it is retro- 
spective and with historical control group [13]. For these reasons the conclusions are less contundent. It is imperative to develop well controlled clinical trials in children to determine the real role of EF in DEBA. The objective of the present study was to assess the tolerability and safety of EF in pediatric patients who underwent DEBA.

\section{Materials and Methods}

The present study was a controlled randomized clinical trial and it was approved by the research and ethics committees of our Hospital and with the ClinicalTrials. gov ID NCT01028807.

\subsection{Inclusion Criteria}

All patients between the ages of 1 month to 18 years old that required elective DEBA (ileum and colon).

\subsection{Exclusion Criteria}

Proximal and non elective anastomosis and high risk groups: newborns, upper gastrointestinal tract anastomosis (esophagus, gastric, duodenal or jejunal), biliousdigestive or rectal anastomosis, immunosuppressed patients, gastrostomy or any pre anastomotic derivation, multiple anastomoses, chronic intestinal obstruction and patients who did not complete the minimum POP follow up of one month. Variables: Demographic: Age, weight, gender, nutritional status, diagnosis. Intraoperative: Surgeons' category, operative time and anastomosis placement.

\subsection{Follow up Tolerance Variables}

Need to insert a NGT, time to start peristalsis and bowel movements, time to full diet intake, post-operative stay.

\subsection{Safety Variables}

Mild and persistent vomiting, persistent abdominal distention, wound infection or dehiscence as well as anastomotic leak or dehiscence, reoperation and death.

\subsection{Development}

Informed consent was obtained from the patient's parents. All patients were managed routinely in the pre operative period with double antibiotic regimen the day of the surgery (clindamicin plus amikacin), bowel preparation with an ethylenglycol based solution in case of colonic anastomosis, and basal measurement of the abdominal girth in the operating room prior to anesthesia administration was taken. Balanced general anesthesia with endotracheal intubation was used in all cases and a Levine type NGT was set in place only during the surgery, all anastomoses were done in one layer with 4-0 polyglactin 910.
Randomization was performed at the end of the procedure through closed envelopes into two groups: 1) Experimental group (EG): Early feeding group: after at least 24 hour of fasting period, and once abdominal conditions were considered appropriate: 1) gas passage through rectum or bowel movements, and 2) without abdominal distention, vomiting or nausea, oral fluids were offered during 24 hour and then advanced to regular diet as tolerated. 2) Control group (CG): Obligatory 5-day fasting, considered as the therapeutic gold standard at our hospital and our country. Both groups were managed with antibiotic regimen, ranitidine and appropriate analgesics but without NGT or antiemetic drugs in either group. Tolerance and safety variables were recorded every 8 hours. Once regular diet was tolerated, the patients were discharged and followed up at clinic 30 days afterwards.

\subsection{Statistics}

Descriptive statistics measurements for global description. Student's t test for quantitative and Chi square test for qualitative variables were used, considering statistically significant a p-value less than 0.05 .

\section{Definitions}

Early feeding: to begin enteral feeding before PO day five. Persistent abdominal distention: Abdominal perimeter increment of $3 \mathrm{~cm}$ or more during $8 \mathrm{hr}$. Persistent vomiting: 3 or more events in less than $24 \mathrm{hr}$.

\section{Results}

Between June 2003 to May 2004 we operated on 92 bowel anastomoses, 32 high-risk that were excluded and 60 elective that were included and its our sample size, thirty within each arm of the study: control and experimental (Figure 1). Both groups were comparable ( $p=N S$ ) in all demographic and intraoperative variables, (age 53 vs 41 months, weight 16.8 vs $15.5 \mathrm{Kg}$, male $70 \%$ vs $63.3 \%$, malnutrition $33 \%$ vs 33\%, colostomy $70 \%$ vs $73 \%$, anorectal malformations $37 \%$ vs $56 \%$, inflammatory $40 \%$ vs 30\%, surgical time 149 vs $136 \mathrm{~min}$ ) showing an homogeneous distribution between both groups (Table 1).

No patient developed vomiting or required insertion of NGT. Eight patients showed mild abdominal distension (less than $3 \mathrm{~cm}$ ) (13\%). Ten had mild fever and 11 had surgical site infection. Four developed minor anastomotic leakage (two in each group) but neither required reoperation or died (Table 2).

Time to start peristalsis, first flatus passage and bowel movement showed no statistical differences between groups and the complication incidence was low and equally distributed $(p=N S)$. There were 11 superficial surgical site infections, none of them with wound dehis- 


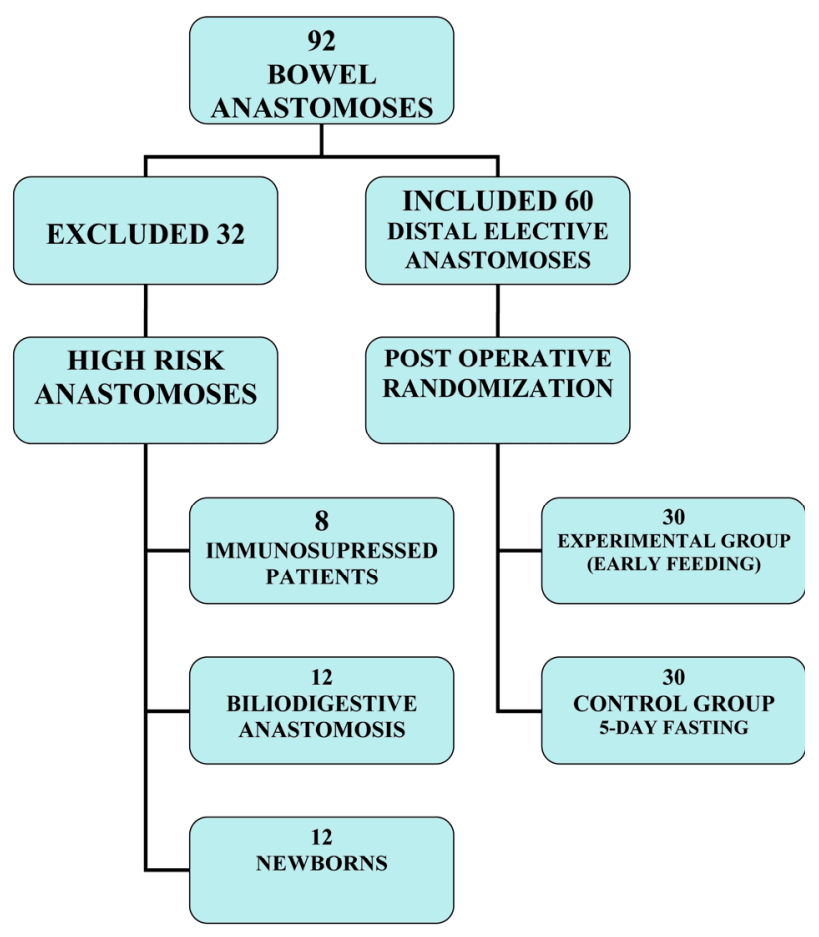

Figure 1. Patient flow chart of the bowel anastomoses operated on between June 2003 and May 2004.

Table 1. Demographic and intra-operative variables and comparison between groups.

\begin{tabular}{cccc}
\hline Variable & $\begin{array}{c}\text { Control group } \\
(\mathrm{n}=30)\end{array}$ & $\begin{array}{c}\text { Experimental group } \\
(\mathrm{n}=30)\end{array}$ & $\mathrm{p}$ \\
\hline Age & $53 \pm 63$ months & $41 \pm 46$ months & 0.08 \\
Weight & $16.8 \pm 15.3 \mathrm{~kg}$. & $15.5 \pm 12.9 \mathrm{~kg}$. & 0.41 \\
Qx time & $149 \pm 51 \mathrm{~min}$ & $136 \pm 31.6 \mathrm{~min}$ & 0.067 \\
Male & $21(70 \%)$ & $19(63.3 \%)$ & 0.78 \\
Malnutrition & $10(33.3 \%)$ & $10(33.3 \%)$ & 0.41 \\
Ethiology & & & \\
ARM 28 (46.7\%) & $11(37 \%)$ & $17(56 \%)$ & \\
HD 8 (13.3\%) & $4(13 \%)$ & $4(13 \%)$ & 0.19 \\
INF 21 (35\%) & $12(40 \%)$ & $9(30 \%)$ & \\
TUM 3 (5\%) & $3(10 \%)$ & 0 & \\
Colostomy & $21(70 \%)$ & $22(73 \%)$ & 0.89 \\
\hline
\end{tabular}

ARM: anorectal malformation, HD: hirschsprung disease, INF: inflammatory disease, TUM: tumoral disease. NS: non significative.

cence and all healed with local antiseptics, 3 in the EG $(10 \%)$ and 8 in the CG $(26.7 \%)(p=0.9)$. Four cases developed mild anastomotic leakage, two in each group (p $=0.69$ ), all healed without surgery. The time to fulfill oral intake was, as expected, earlier in the EG (2nd POP day vs 5 POP day in the CG $(p=0.001)$ ) and also the POP stay was lower in the EG than in the CG $(6.0 \pm 2.9$ vs $9.8 \pm 4.1$ days) but didn't reach statistical significance (p = 0.08) (Table 2).
Table 2. Follow-up variables. Tolerance and safety variables and its comparison among groups.

\begin{tabular}{cccc}
\hline Variable & $\begin{array}{c}\text { Control group } \\
(\mathrm{n}=30)\end{array}$ & $\begin{array}{c}\text { Experimental group } \\
(\mathrm{n}=30)\end{array}$ & $\mathrm{p}$ \\
\hline Peristalsis & $1.87 \pm 0.6$ days & $1.43 \pm 0.5$ days & 0.71 \\
Flatus & $2.13 \pm 0.5$ days & $1.63 \pm 0.5$ days & 0.21 \\
Bowel movement & $2.6 \pm 0.56$ days & $1.63 \pm 0.55$ days & 0.68 \\
Full diet & $5.0 \pm 0$ days & $2.1 \pm 0.3$ days & 0.001 \\
Distension & $4(13.3 \%)$ & $4(13.3 \%)$ & 0.64 \\
Fever & $7(23.3 \%)$ & $3(10 \%)$ & 0.15 \\
Wound infection & $8(26.7 \%)$ & $3(10 \%)$ & 0.9 \\
Leakage & $2(6.7 \%)$ & $2(6.7 \%)$ & 0.69 \\
Hospital stay & $9.8 \pm 4.1$ days & $6.0 \pm 2.9$ days & 0.08 \\
\hline
\end{tabular}

\section{Discussion}

Our results showed that both groups were comparable given the homogeneity in the distribution of the demographic and intraoperative variables, and therefore, the only difference was the early feeding in the experimental group. This is in contrast to previous studies that lacked an adequate methodology for their series of children by being retrospective and having an historical control group [13]. Our results also demonstrated that both groups had an identical POP course because the tolerability and safety variables showed no difference. There were two limitations of the study: First it was not blinded for the personel that recorded the follow up variables because it was impossible to hide the food in the control group and second, it only included distal elective bowel anastomosis (ileum and colon), so there is a need of more studies to test the safety on other types of anastomosis. Although it was not a blinded study, our observations support early feeding in distal elective bowel anastomosis in children. Peristalsis, first flatus passage and bowel movements began earlier in the EG, however, it did not reach statistical significance (Table 2). It is possible that with a bigger sample these differences will become apparent.

In our study, the 5-day fasting did not confer any preventive role to avoid POP complications given that the frequency of persistent abdominal distention, vomiting, infection or dehiscence of the surgical wound, dehiscence of the intestinal anastomosis, entero-cutaneous fistulae and reoperations were similar in both groups $(\mathrm{p}=$ NS). All this supports an early feeding policy among DEBA in pediatric patients. This is in concordance to a systematic review of randomized clinical trials concluding that even when the individual clinical complications failed to reach statistical significance, the trend is that EF may reduce the risk of POP complications in adult pa- 
tients $[4,12]$.

The observed results in this series of patients demonstrated a shorter POP hospital stay in the experimental group but this difference did not reach statistical significance ( $p=0.08)$. Such observations might be due to the fact that at the beginning of the study, a few patients in the EG were kept in the hospital for a longer period of time for observation-at least two additional days, on average. This was primarily due to concerns related to the risk of missing post-operative complications. Patients enrolled later in the EG were discharged as soon as bowel function returned to normal. For that reason, the shorter hospital stay observed in the patients enrolled in the EG appeared to be of clinical significance but it did not reach statistical significance.

\section{Conclusion}

We consider that this study supports scientifically that there is no necessity to keep the 5-day fasting in order to prevent post operative complications among children with DEBA, and therefore should not be used routinely. Based on the results of this study, it can be inferred that prolonged fasting in DEBA patients can be avoided. This is important since post-operative fasting combined with the surgical stress response may contribute to nosocomial infections, surgical site infections, acute malnutrition, and distress related to the starving sensation. Our observations may improve the quality of post-operative care of children after gastrointestinal surgery.

\section{REFERENCES}

[1] R. Davila-Perez, E. Bracho-Blanchet, J. M. Tovilla-Mercado, J. A. Hernandez-Plata, A. Reyes and J. Nieto-Zermeño, "Unnecessary Gastric Decompression in Distal Elective Bowel Anastomoses in Children. A Randomized Study," World Journal of Surgery, Vol. 34, No. 5, 2010, pp. 947-953. doi:10.1007/s00268-010-0442-3

[2] M. L. Pearl, F. A. Valea, M. Fisher, L. Mahler and E. Chalas, "A Randomized Controlled Trial of Early Postoperative Feeding in Gynecologic Oncology Patients Undergoing Intra-Abdominal Surgery,” Obstetrics \& Gynecology, Vol. 92, No. 1, 1998, pp. 94-97. doi:10.1016/S0029-7844(98)00114-8

[3] B. T. Stewart, R. J. Woods, B. T. Collopy, R. J. Fink, J. R. Mackay and J. O. Keck, "Early Feeding after Elective Open Colorectal Resections: A Prospective Randomized Trial,” Australian and New Zealand Journal of Surgery, Vol. 68, No. 2, 1998, pp. 125-128. doi:10.1111/j.1445-2197.1998.tb04721.x
[4] I. J. Han-Geurts, W. C. J. Hop, N. F. M. Kok, et al., "Randomized Clinical Trial of the Impact of Early Enteral Feeding on Postoperative Ileus and Recovery," British Journal of Surgery, Vol. 94, No. 5, 2007, pp. 555-561. doi:10.1002/bjs.5753

[5] I. Gokpinar, E. Gurleyik, M. Pehlivan, O. Ozcan, I. Ozaydin, A. Aslaner, Y. Demiraran and M. Gultepe, "Early Enteral and Glutamine Enriched Enteral Feeding Ameliorates Healing of Colonic Anastomosis: Experimental Study," Ulusal Travma ve Acil Cerrahi Dergisi, Vol. 12, No. 1, 2006, pp. 17-21.

[6] P. Reissman, T. A. Teoh, S. M. Cohen, E. G. Weiss, J. J. Nogueras and S. D. Wexner, "Is Early Oral Feeding Safe after Colorectal Surgery? A Prospective Randomized Trial,” Annals of Surgery, Vol. 222, No. 1, 1995, pp. 73- 77. doi:10.1097/00000658-199507000-00012

[7] V. Seenu and A. K. Goel, "Early Oral Feeding after Elective Colorectal Surgery: Is It Safe,” Tropical Gastroenterology, Vol. 16, No. 4, 1995, pp. 72-73.

[8] M. Senkal, A. Mumme, U. Eickhoff, B. Geier, G. Spath, D. Wulfert, U. Joosten, A. Frei and M. Kemen, "Early Postoperative Enteral Immunonutrition: Clinical Outcome and Cost Comparison Analysis in Surgical Patients," Critical Care Medicine, Vol. 25, No. 9, 1997, pp. 14891496. doi:10.1097/00003246-199709000-00015

[9] T. Bisgaard and H. Kehlet, "Early Oral Feeding after Elective Abdominal Surgery-What Are the Issues?” $\mathrm{Nu}$ trition, Vol. 18, No. 11, 2002, pp. 944-948. doi:10.1016/S0899-9007(02)00990-5

[10] C. V. Feo, B. Romanini, D. Sortini, R. Ragazzi, P. Zamboni, G. C. Pansini and A. Liboni, "Early Oral Feeding after Colorectal Resection: A Randomized Controlled Study,” ANZ Journal of Surgery, Vol. 74, No. 5, 2004, pp. 298-301. doi:10.1111/j.1445-1433.2004.02985.x

[11] P. A. Lucha Jr., R. Butler, J. Plichta and M. Francis, “The Economic Impact or Early Enteral Feeding in Gastrointestinal Surgery: A Prospective Survey of 51 Consecutive Patients,” American Surgeon, Vol. 71, No. 3, 2005, pp. 187-190.

[12] H. K. Andersen, S. J. Lewis and S. Thomas, "Early Enteral Nutrition within 24 h of Colorectal Surgery versus Later Commencement of Feeding for Postoperative Complications (Review)," Cochrane Collaboration, Vol. 2, 2009, pp. 1-30.

[13] S. Sangkhathat, S. Patrapinyokul and K. Tadyathikom, "Early Enteral Feeding after Closure of Colostomy in Pediatric Patients," Journal of Pediatric Surgery, Vol. 38, No. 10, 2003, pp. 1516-1519. doi:10.1016/S0022-3468(03)00506-2 